ARTICLE

\title{
Large-scale wet-spinning of highly electroconductive MXene fibers
}

\author{
Wonsik Eom (1) 1,3, Hwansoo Shin (10) 1,3, Rohan B. Ambade ${ }^{1,3}$, Sang Hoon Lee (1) 1, Ki Hyun Lee (D) 1,2, \\ Dong Jun Kang ${ }^{1} \&$ Tae Hee Han (1) ${ }^{1,2}$ 遖
}

$\mathrm{Ti}_{3} \mathrm{C}_{2} \mathrm{~T}_{x}$ MXene is an emerging class of two-dimensional nanomaterials with exceptional electroconductivity and electrochemical properties, and is promising in the manufacturing of multifunctional macroscopic materials and nanomaterials. Herein, we develop a straightforward, continuously controlled, additive/binder-free method to fabricate pure MXene fibers via a large-scale wet-spinning assembly. Our MXene sheets (with an average lateral size of $5.11 \mu \mathrm{m}^{2}$ ) are highly concentrated in water and do not form aggregates or undergo phase separation. Introducing ammonium ions during the coagulation process successfully assembles $M X e n e$ sheets into flexible, meter-long fibers with very high electrical conductivity $\left(7,713 \mathrm{~S} \mathrm{~cm}^{-1}\right)$. The fabricated MXene fibers are comprehensively integrated by using them in electrical wires to switch on a light-emitting diode light and transmit electrical signals to earphones to demonstrate their application in electrical devices. Our wet-spinning strategy provides an approach for continuous mass production of MXene fibers for high-performance, next-generation, and wearable electronic devices.

\footnotetext{
${ }^{1}$ Department of Organic and Nano Engineering, Hanyang University, Seoul 04763, Republic of Korea. ${ }^{2}$ Research Institute of Industrial Science, Hanyang University, Seoul 04763, Republic of Korea. ${ }^{3}$ These authors contributed equally: Wonsik Eom, Hwansoo Shin, Rohan B. Ambade. ${ }^{凶}$ email: than@ @anyang.ac.kr
} 
$\mathrm{T}$ wo-dimensional (2D) nanosheets with fascinating properties are building blocks for potential applications ${ }^{1,2}$. Compared with their bulk counterparts, 2D nanomaterials are easy-to-assemble structures for nanoscale architectures that have appealing electronic, chemical, physical, and mechanical properties; a high specific surface area; and versatile surface chemistries $^{3-5}$. To date, various $2 \mathrm{D}$ materials, such as graphene, hexagonal boron nitride (h-BN), graphitic carbon nitride ( $\mathrm{g}$ $\mathrm{C}_{3} \mathrm{~N}_{4}$ ), transition metal dichalcogenides (TMDs), black phosphorus (BP), and transition metal oxides (TMOs), have garnered considerable attention, and many strategies have been proposed to develop them into macroscopic structures ${ }^{6-10}$. For example, significant advancements have been made regarding the development of macroscopic one-dimensional (1D) carbon-based fibers prepared from graphene oxides $(\mathrm{GO})^{11}$. Graphene-related fibers have gained considerable interest because of their versatile functionalities, such as lightweight, mechanical flexibility, bendability, stretchability, and the ability to be woven into textiles for the next generation of smart electronic gadgets ${ }^{12,13}$. In particular, to realize the macroscopic assembly of $2 \mathrm{D}$ nanosheets into fiber structures, the wet-spinning process, which utilizes the phase change ability of highly concentrated colloidal dispersions (i.e., $\backslash$ in the liquid state) to transform into gel-fiber assemblies and solid fibers in a coagulation bath, has been demonstrated as a versatile pathway for the long and continuous mass production of fibers ${ }^{11,14}$. Notably, understanding the molecular interaction between sheets and systematic studies on the parameters of the coagulation process are crucial to achieving fiber formation from individual colloidal particles.

$\mathrm{Ti}_{3} \mathrm{C}_{2} \mathrm{~T}_{x}$ MXene is composed of transition metal nitrides and carbides (MXenes) and has been extensively explored as an emerging family of 2D materials because of its excellent electrical-thermal conductivity, mechanical, and chemical properties, and wide range of potential applications ${ }^{15-19}$. MXenes have the general configuration of $M_{n+1} X_{n} T_{x}$, in which $M, X$, and $T$ represent transition metals, carbon/nitrogen, and surface terminal functionalities, such as $\mathrm{O}, \mathrm{F}$, and $\mathrm{OH}$, respectively. MXenes are typically obtained as sheet materials with a nanoscale thickness via the delamination of the MAX $\left(M_{n+1} A X_{n}\right)$ phase $^{16}$. Very recently, several researchers have attempted to fabricate MXenebased fibers using wet-spinning and electrospinning with MXene/ polymer blend dope solutions and MXene/rGO for coassembly ${ }^{20,21}$. However, the intrinsically high electrical conductivity of pure $\mathrm{Ti}_{3} \mathrm{C}_{2} \mathrm{~T}_{x}$ MXene (up to $9880 \mathrm{~S} \mathrm{~cm}^{-1}$ for spraycast films) is lower than that of MXene composites with reduced GO (rGO) $\left(72-290 \mathrm{~S} \mathrm{~cm}^{-1}\right)^{21,22}$, CNT fiber $\left(26 \mathrm{~S} \mathrm{~cm}^{-1}\right)^{23}$, and PEDOT:PSS $\left(1489 \mathrm{~S} \mathrm{~cm}^{-1}\right)^{24}$, showing that the conductivity of MXenes is not fully utilized in the fiber form. The crucial challenge regarding wet-spinning for pure MXene fibers is the weak self-supporting organization because of poor interlayer interaction between the relatively small MXene sheets. In addition, a low concentration of dispersion has made it challenging to process MXene directly into a $1 \mathrm{D}$ fiber form.

Herein, we report a straightforward and reliable synthetic route for continuously controlled fabrication of additive/binder-free, composite-free, entirely pure 1D MXene fibers with high electrical conductivity by a wet-spinning assembly (Fig. 1). The dispersion with a relatively large $\mathrm{Ti}_{3} \mathrm{C}_{2} \mathrm{~T}_{x}$ MXene sheet (the average size and aspect ratio were $\sim 5.11 \mu \mathrm{m}^{2}$ and 1600 , respectively) at a high concentration $\left(25 \mathrm{mg} \mathrm{mL}^{-1}\right)$ demonstrated highly stable colloidal properties in a lyotropic liquid-crystalline phase. The wet-spinning of the $2 \mathrm{D}$ MXene spinning dope successfully produced flexible meter-long continuous MXene fibers with an ultrahigh electrical conductivity of $7713 \mathrm{~S} \mathrm{~cm}^{-1}$. The MXene fibers that exhibited excellent performance were used for the electrical wires to switch on an LED light and wires to transmit electrical signals to earphones. Furthermore, the MXene fibers showed high flexibility and excellent mechanical properties. The wet-spinning strategy reported in this work suggests a method for the continuous mass production of MXene fibers, which indicates that they are promising candidates for high-performance, flexible, portable, and wearable electronics. The development of nanoscale properties on the macroscopic level using a scalable assembly represents progress toward the practical application of these extraordinary $2 \mathrm{D}$ materials.

\section{Results}

Synthesis and characterization of $\mathrm{Ti}_{3} \mathrm{C}_{2} \mathrm{~T}_{x}$ MXene sheets. A MAX-phase $\left(\mathrm{Ti}_{3} \mathrm{AlC}_{2}\right)$ powder with graphite-like stacked-layer structures was observed in images obtained via scanning electron microscopy (SEM) (Fig. 2a and Supplementary Fig. 1a). MXene $\left(\mathrm{Ti}_{3} \mathrm{C}_{2} \mathrm{~T}_{x}\right)$ sheets were obtained by selectively etching the $\mathrm{Al}$ from a $\mathrm{Ti}_{3} \mathrm{AlC}_{2}$ powder using $\mathrm{LiF}$ and $\mathrm{HCl}$, as reported previously ${ }^{25}$ SEM images of fully exfoliated MXene monolayers showed an average lateral size of $2.26 \pm 0.95 \mu \mathrm{m}$ (Fig. $2 \mathrm{~b}$ and Supplementary Fig. $1 \mathrm{~b}, \mathrm{c})$. The height profile obtained via atomic force microscopy (AFM) mapping revealed that the MXene sheets had a height of $1.35-1.81 \mathrm{~nm}$, which corresponded to a single layer of the MXene, implying the successful exfoliation of the sheets (Supplementary Fig. 1d and e) ${ }^{26}$. The folded MXene was identified as a double layer according to the AFM height profile, which showed a height of 3.31-3.72 nm; this agrees with the results of previous reports ${ }^{26}$. Conductive atomic force microscopy (CAFM) clearly showed that the MXene sheets were very electrically conductive (Fig. 2d, e). The prepared MXene monolayer was also observed using transmission electron microscopy (TEM), and highly crystalline lattice fringes with a lattice spacing of $0.26 \mathrm{~nm}$ corresponding to the $\mathrm{Ti}_{3} \mathrm{C}_{2} \mathrm{~T}_{x}$ (100) plane were clearly observed in the HR-TEM images. The selected area electron diffraction (SAED) pattern confirmed that the MXene sheets had a typical hexagonal symmetry (Supplementary Fig. $2 a$ and $b$ ). The XRD pattern and the atomic percent of MXene confirmed the complete etching of the Al layer (Supplementary Fig. $2 \mathrm{c}$ and Supplementary Table 1$)^{27}$. The corresponding elemental maps confirmed the uniform distribution of all elements, suggesting that the surface of the MXene sheet contained oxygen and fluorine as termination groups (Supplementary Fig. 2d).

The chemical functionalities of the exfoliated MXene sheets were further examined via X-ray photoelectron spectroscopy (XPS). The deconvoluted $\mathrm{C} 1 s, \mathrm{O} 1 s$, and Ti2p XPS peaks demonstrated that inherent termination groups, such as $\mathrm{C}-\mathrm{Ti}-\mathrm{T}_{x}$, $\mathrm{C}-\mathrm{Ti}-(\mathrm{OH})_{x}$, and $\mathrm{C}-\mathrm{Ti}-\mathrm{O}_{x}$, existed on the surface of the MXene and were likely introduced during the $\mathrm{Al}$ etching of the MAX crystals (Fig. 3a, b, Supplementary Fig. 2a-c). Notably, these surface functionalities are important for the formation of a stable dispersion in an aqueous medium ${ }^{28}$. The negative surface charge values increased with the $\mathrm{pH}$ of MXene, owing to the ionizable surface termination groups, suggesting strong electrostatic repulsion between the adjacent sheets (Fig. 3c) ${ }^{29,30}$. The apparent dispersibility of the MXene was observed at various concentration ranges (Fig. 3d), and no sediment was formed on the bottom of the vials at different MXene concentrations. The absorbance of the MXene dispersion was examined, as shown in Supplementary Fig. 3d. In the inset of Supplementary Fig. 3d, a linear relationship between the UV absorbance and concentration of MXene sheets was observed, confirming the stability of the dispersed state ${ }^{31}$.

Rheological properties of MXene ink. At a high concentration $\left(25 \mathrm{mg} \mathrm{mL}^{-1}\right)$, the MXene dispersion formed a viscous ink with a viscosity of $3.87 \times 10^{3} \mathrm{Pas}$ and without aggregates and phase separation of solid particles and dispersing media (Fig. 3e) ) $^{28,32}$ 


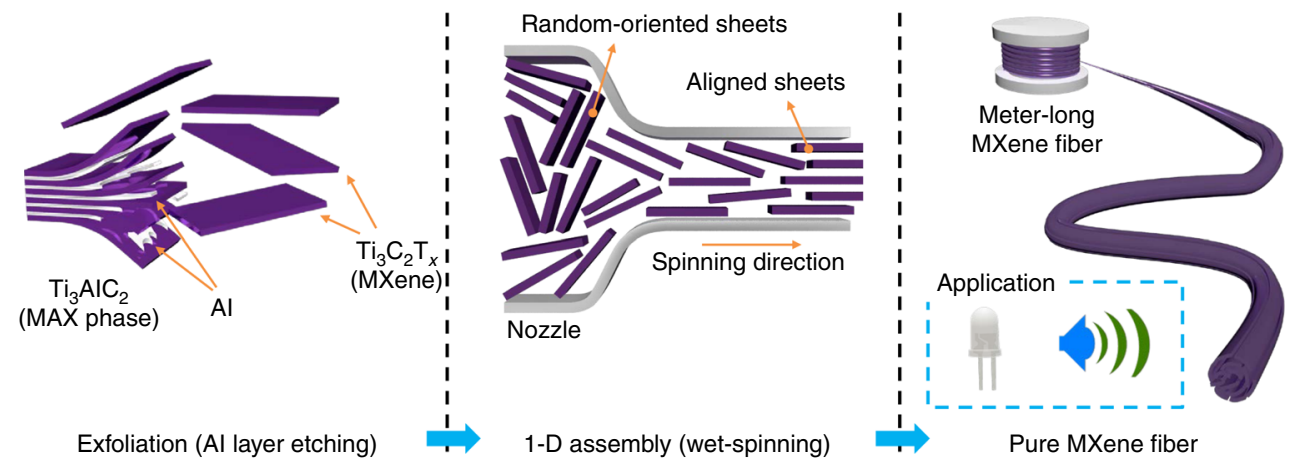

Fig. 1 Schematic illustration of the reconstruction of $\mathbf{M X e n e}$ single layers into $\mathbf{M X e n e}$ fibers. $\mathrm{Ti}_{3} \mathrm{AlC}_{2}$ was exfoliated to $\mathrm{Ti}_{3} \mathrm{C}_{2} T_{x}$ by etching the $A l$ layer. The MXene was highly concentrated in the aqueous dispersion and assembled into a fiber, which was aligned in the axial direction by the wet-spinning process. The obtained pure MXene fiber was used for electric energy and signal transfer applications.
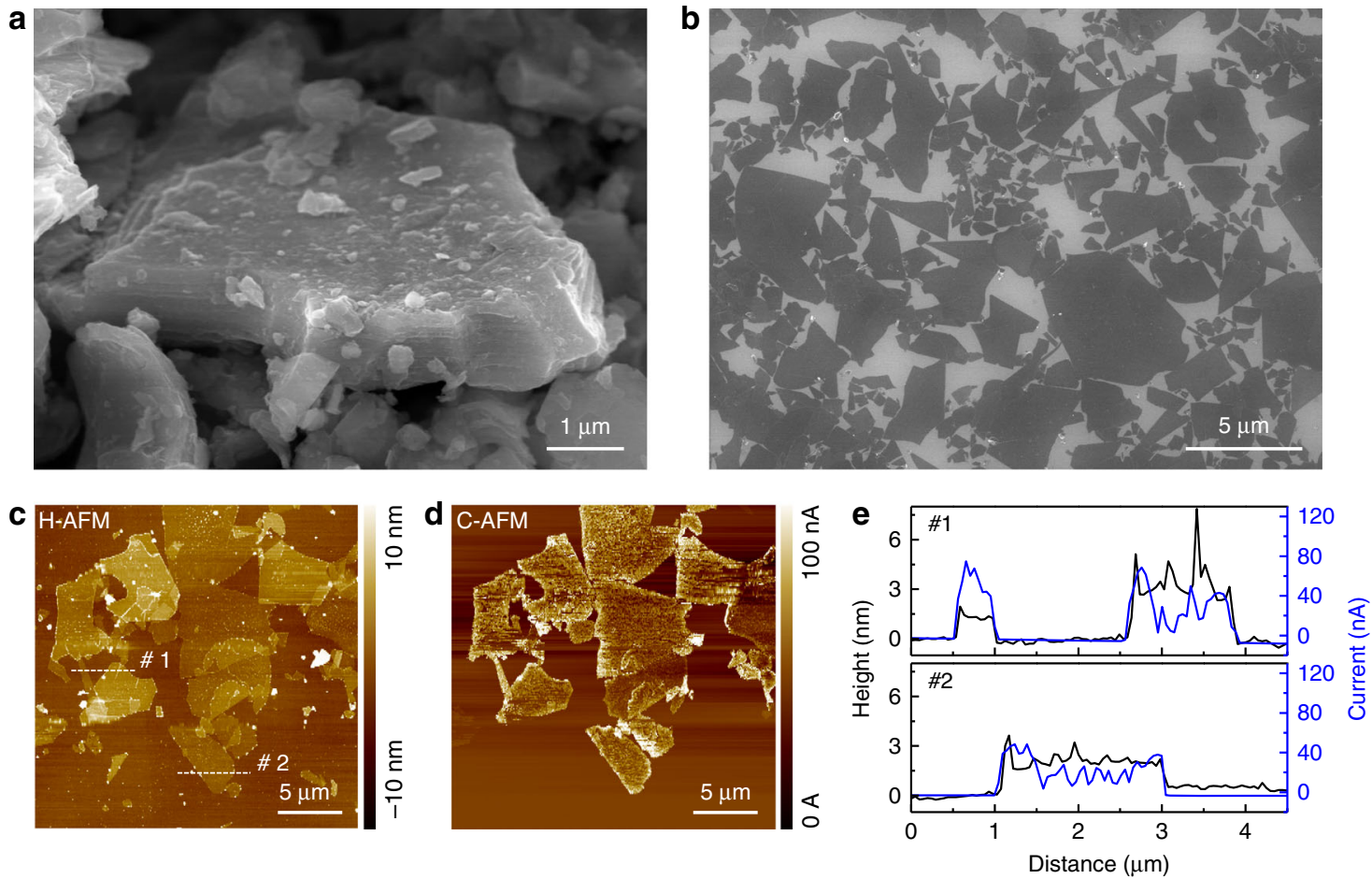

Fig. 2 Synthesis and identification of $\mathbf{T i}_{3} \mathbf{C}_{\mathbf{2}} \mathbf{T}_{\mathbf{x}}$ (MXene). Morphology of a MAX-phase particle and $\mathbf{b} M X e n e$ single layer coated on a SiO ${ }_{2}$ wafer. $\mathbf{c} A F M$ and $\mathbf{d}$ C-AFM images of MXene single sheets in the same area. $\mathbf{e}$ Height and current-line profile of lines \#1 and \#2 in $\mathbf{c}$

Based on Onsager's theoretical prediction model, MXene sheets can exhibit lyotropic liquid-crystalline properties from $\sim 16 \mathrm{mg} \mathrm{mL}^{-133}$. As shown in Fig. 3f, the MXene dispersion $\left(25 \mathrm{mg} \mathrm{mL}^{-1}\right)$ also exhibited birefringence between two crossed polarizers, indicating the formation of a liquid-crystalline phase as a result of local orientation that did not aggregate. As is often observed in complex fluid systems containing rigid polymer chains, the viscosity of the MXene increased with the concentration and decreased with an increase in the shear rate

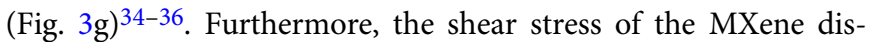
persion significantly decreased at the initial stage and then gradually increased with the shear rate (Fig. $3 \mathrm{~h}$ ), suggesting that the randomly oriented MXene sheets became arranged as a result of the shear-induced deformation ${ }^{10}$. A decrease in the shear stress was clear in concentrated dispersions (higher than $15 \mathrm{mg} \mathrm{mL}^{-1}$ ). Notably, the ratio of the storage modulus to the loss modulus $\left(G^{\prime} / G^{\prime \prime}\right)$ of a dispersion can be used as an indicator for the spinnability of liquid-crystalline 2D colloidal dispersions ${ }^{37}$. Wallace et al. reported that the wet-spinning of GO dispersions is achievable when the value of $G^{\prime} / G^{\prime \prime}$ is between 1.80 and 6.36 at an angular rate of $0.02 \mathrm{~Hz}$. Gogotsi et al. also expected that highly concentrated MXene dispersions can be wet-spun into fibers by following this relation ${ }^{35}$. Similarly, we found that the spinnability of an MXene dispersion could be predicted using the $G^{\prime} / G^{\prime \prime}$ value of the MXene. Experimentally, at $5 \mathrm{mg} \mathrm{mL}^{-1}$, the MXene dispersion herein was not capable of forming fibers because of the weak gel strength, and the value of $G^{\prime} / G^{\prime \prime}$ was 13.33 (Supplementary Fig. 4). When the $G^{\prime} / G^{\prime \prime}$ value of the MXene was 6.64 at $12 \mathrm{mg} \mathrm{mL}^{-1}$, the fiber was not stably formed herein, but MXene dispersions with over $15 \mathrm{mg} \mathrm{mL}^{-1}$ (the $G^{\prime} / G^{\prime \prime}$ value was 5.29 at $15 \mathrm{mg} \mathrm{mL}^{-1}$ ) were successfully fabricated into MXene fibers (Fig. 3i).

Wet-spinning of pure MXene fibers. Note that the colloidal stability of MXene sheets can be considerably affected by salts. The role of $\mathrm{NH}_{4}$ ions in the gelation of MXene dispersions was confirmed by the vial inversion method (Fig. 4a) ${ }^{14}$. Indeed, similar to the behavior of graphene and other $2 \mathrm{D}$ materials, the 
a

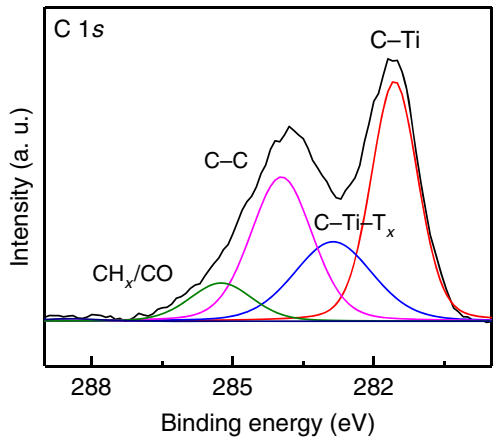

d
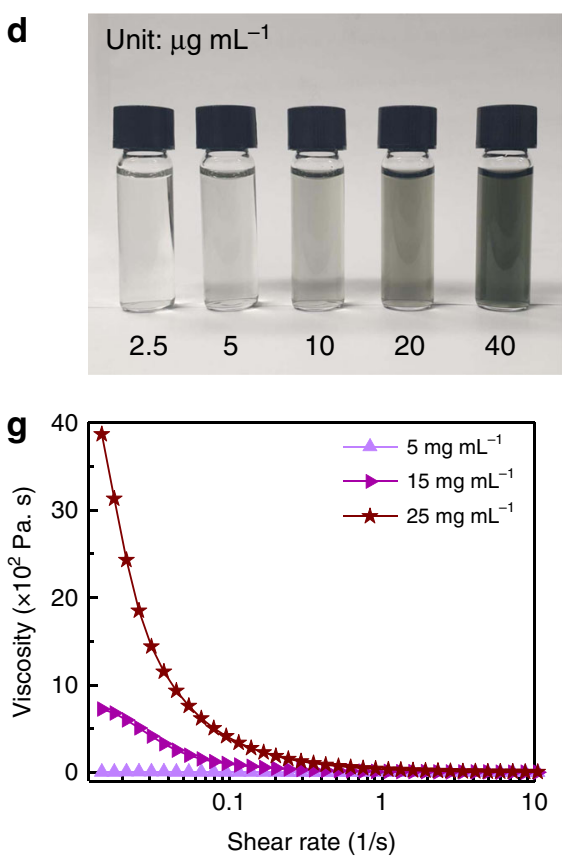

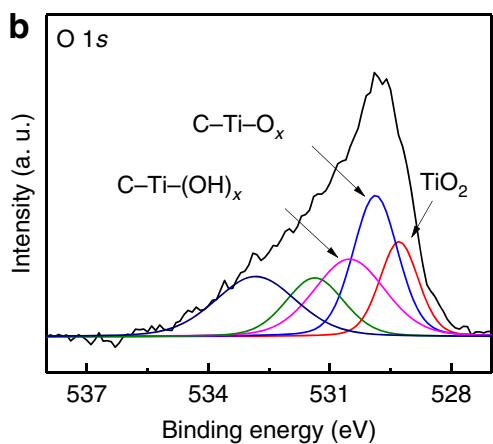

e $25 \mathrm{mg} \mathrm{mL}^{-1}$

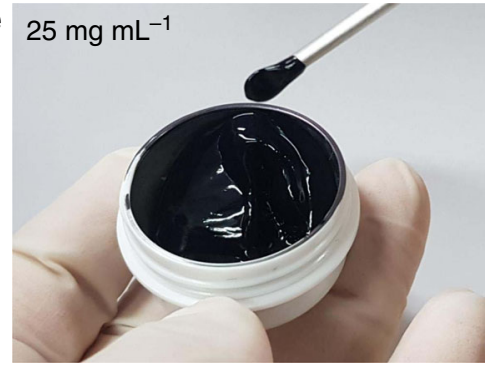

h

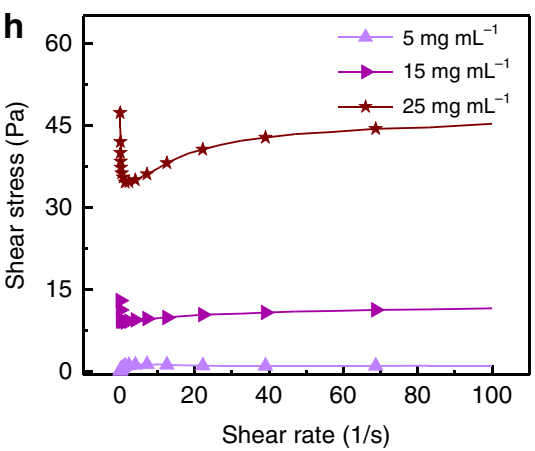

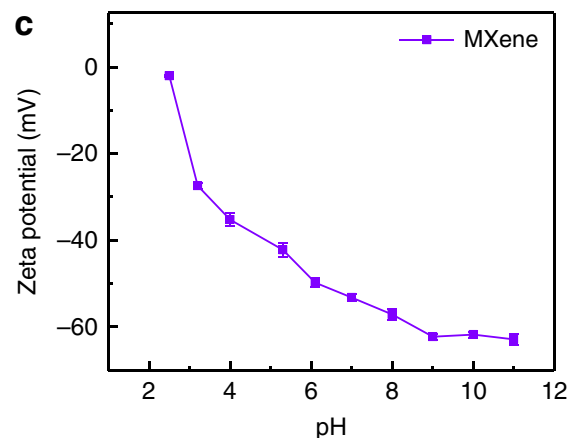
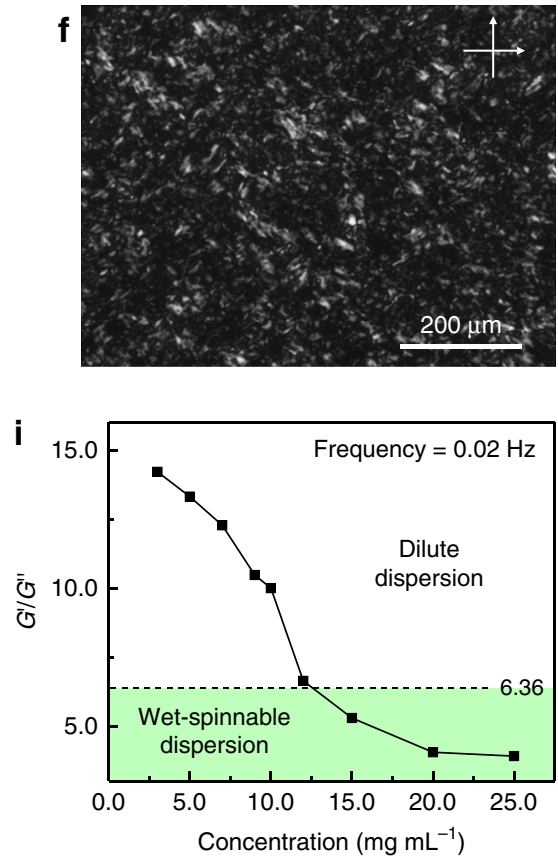

Fig. 3 Dispersibility and spinnability of $\mathbf{M X e n e}$ nanosheets in liquid-crystalline dispersion. a C $1 \mathrm{~s}$ and $\mathbf{b} \mathrm{O} 1 \mathrm{~s}$ XPS spectra of MXene obtained from the dispersion. c Zeta potential of the $\mathrm{MXene}$ as a function of $\mathrm{pH}$ in the aqueous dispersions at a concentration of $\sim 0.05 \mathrm{mg} \mathrm{mL}^{-1}$. $\mathbf{d}$ Optical image of diluted MXene dispersions with concentrations of $0.0025,0.005,0.01,0.02$, and $0.04 \mathrm{mg} \mathrm{mL}^{-1}$ at $755 \mathrm{~nm}$. e Optical image of the concentrated $\mathrm{MXene}$ LC

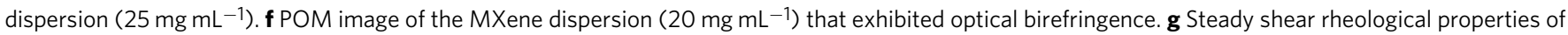

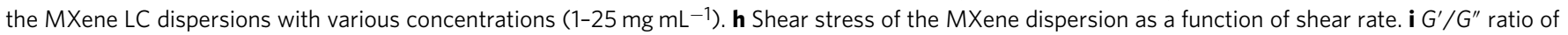
the $M X e n e$ dispersion as a function of concentration. The green region under $G^{\prime} / G^{\prime \prime}=6.36$ indicates the region for the wet-spinning of the $M X$ Xene dispersion at a specific shear rate $(0.02 \mathrm{~Hz})$.

high degree of exfoliation/delamination and gelation of MXenes is essential for continuous fiber fabrication. The prepared MXene liquid crystal dispersion was extruded into a coagulation solution with $\mathrm{NH}_{4}$ ions and then washed in a water bath through a reel to produce continuous fibers using a simple wet-spinning method (Fig. $4 \mathrm{~b}$ and Supplementary video). The extruded MXene did not form gel fibers without $\mathrm{NH}_{4}$ ions (Supplementary Fig. 5). Finally, the fibers were dried in air for $24 \mathrm{~h}$ and formed uniform, long, continuous MXene fibers oriented in the axial direction. The meterlong MXene fibers produced on a large scale by continuous spinning were wound onto a bobbin (Fig. 4c). The extruded 100\% pure MXene fibers that were longer than $1 \mathrm{~m}$ were stable with continuous spinning (Fig. 4d). The cross-section of the MXene fibers showed a lamellar structure with highly compact nanosheets (Fig. $4 \mathrm{e}-\mathrm{g}$ ). The rugged morphology on the side of the fibers indicated that drying and shrinking occurred (Fig. 4g). Highly conducting MXene fibers were used for an electrical application that involved successfully switching on a white light-emitting diode (LED) light (Fig. 4h). Furthermore, the MXene fibers replaced commercially used wires and were integrated into earphone wires to transmit electrical signals (Fig. 4i, Supplementary audio).
Performances of MXene fiber. Figure 5 compares the electrical conductivity and Young's modulus of our fabricated MXene fibers with those of MXene hybrid fibers and graphene fibers fabricated in previous studies (Supplementary Fig. 6 and Supplementary Table 2$)^{21-24,38-45}$. From the Ashby plot, it is clearthat our wet-spun pure MXene fibers are superior to the other considered fibers in terms of electrical conductivity and Young's modulus. The electrical conductivity of the MXene fibers $\left(7713 \mathrm{~S} \mathrm{~cm}^{-1}\right)$ was almost 107 and 27 times higher than that of MXene/graphene hybrid fibers $\left(72.3\right.$ and $290 \mathrm{~S} \mathrm{~cm}^{-1}$, respectively) $)^{21,22}$ and five times higher than that of MXene/PEDOT:PSS fibers $\left(1490 \mathrm{~S} \mathrm{~cm}^{-1}\right)^{24}$, which were reported previously. Furthermore, the conductivity of the MXene fibers in this work was 12-220 times higher than that of the graphene fibers ${ }^{38-44}$. In addition, the pure MXene fibers were 3.2 times more conductive than reported MXene films at the macroscopic scale, implying that the MXene fibers had a well-constructed structure ${ }^{45}$.

\section{Discussion}

We effectively developed pure $\mathrm{Ti}_{3} \mathrm{C}_{2} \mathrm{~T}_{x}$ MXene fibers that were free of additives/binders or composites via a straightforward, 

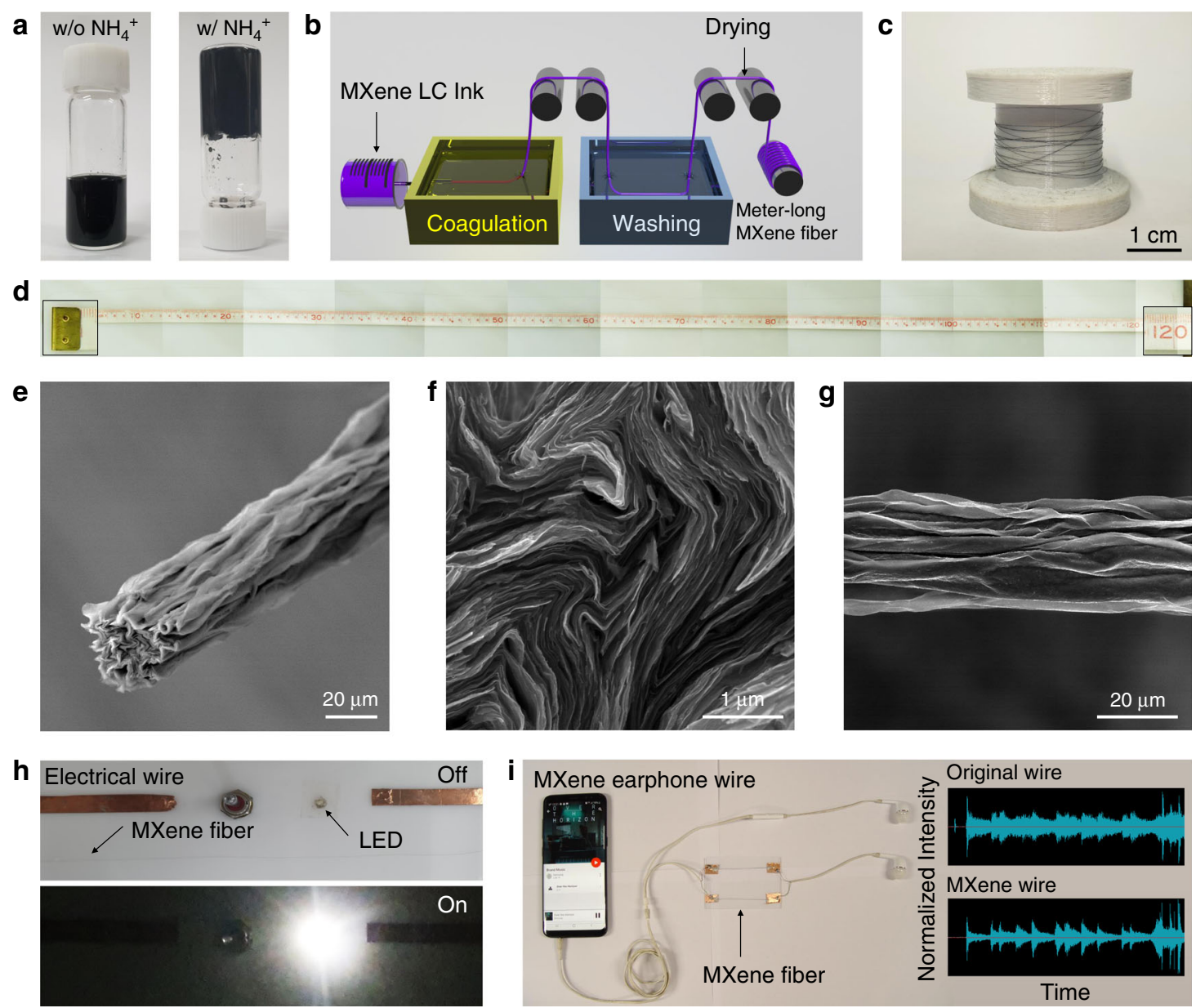

Fig. 4 Wet-spinning of the pure $\mathbf{T i}_{\mathbf{3}} \mathbf{C}_{\mathbf{2}} \mathbf{T}_{\mathbf{x}} \mathbf{M X e n e}$ fiber. a Gelation of the $\mathrm{Ti}_{3} \mathrm{C}_{2} \mathrm{~T}_{\mathbf{x}} \mathrm{MXene}$ dispersion by $\mathrm{NH}_{4}+$ ions. The sol-gel transition was identified via the vial-inversion method. $\mathbf{b}$ Schematic illustration of the wet-spinning process of the $\mathrm{Ti}_{3} \mathrm{C}_{2} \mathrm{~T}_{x} \mathrm{MXene}$ fiber. $\mathbf{c}$ Meter-long $\mathrm{Ti}_{3} \mathrm{C}_{2} \mathrm{~T}_{x} M X e n e$ fiber wound on the bobbin. $\mathbf{d}$ Continuous wet-spinning allowed fabrication of over $1 \mathrm{~m}(1.2 \mathrm{~m})$ of the $\mathrm{Ti}_{3} \mathrm{C}_{2} T_{x} M X$ ene fiber. SEM images of $\mathrm{Ti}_{3} C_{2} T_{x} M X e n e$ fiber: e overall, $\mathbf{f}$ in cross-section, and $\mathbf{g}$ side-section views. $\mathrm{Ti}_{3} \mathrm{C}_{2} \mathrm{~T}_{x} \mathrm{MXene}$ fiber applied as $\mathbf{h}$ an electrical wire and $\mathbf{i}$ an earphone wire.

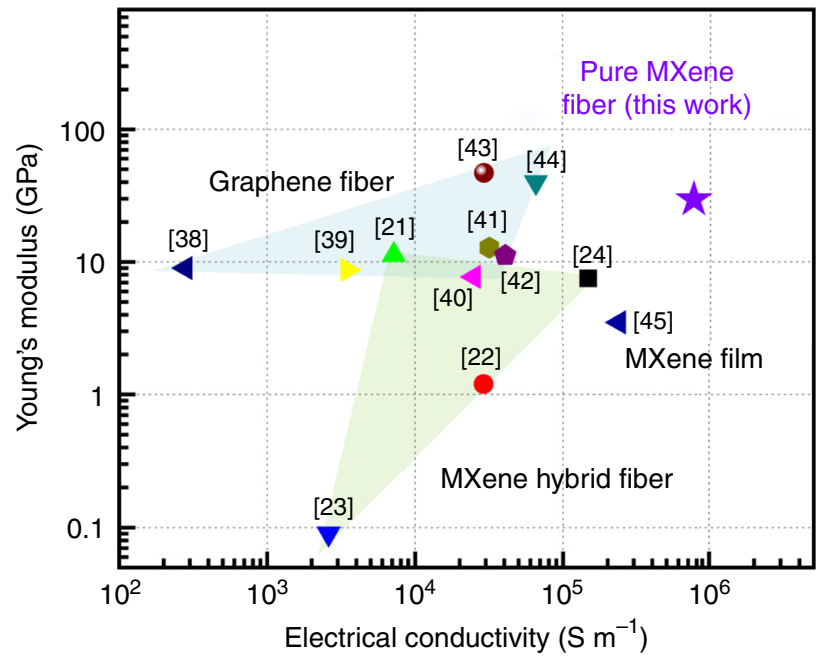

Fig. 5 Comparison of electrical conductivity and Young's modulus. The MXene fiber is compared with previous graphene fibers and MXene hybrid fibers.

continuous, large-scale, wet-spinning strategy. The large $\mathrm{Ti}_{3} \mathrm{C}_{2} \mathrm{~T}_{x}$ MXene sheets had an excellent dispersion at a high concentration of $25 \mathrm{mg} \mathrm{mL}^{-1}$ and demonstrated liquid-crystalline patterns and rheological properties of lyotropic liquid crystals. The $\mathrm{Ti}_{3} \mathrm{C}_{2} \mathrm{~T}_{x}$
MXene fibers fabricated by a wet-spinning method herein exhibited a very high electrical conductivity of $7713 \mathrm{~S} \mathrm{~cm}^{-1}$, and flexible, continuous, meter-long MXene fibers were successfully fabricated. Considering these outstanding properties, we comprehensively integrated the $\mathrm{Ti}_{3} \mathrm{C}_{2} \mathrm{~T}_{x}$ MXene fibers in electrical wires for switching on an LED light and transmitting electrical signals to earphones to demonstrate the application of the fibers in miniaturized portable devices. Therefore, we believe that our wet-spinning strategy for continuous mass production of pure $\mathrm{Ti}_{3} \mathrm{C}_{2} \mathrm{~T}_{x}$ MXene fibers offers a way to exploit the original nanoscale potential of MXenes at the macroscopic scale. In addition, the approach herein advances the use of a large family of MXenes in next-generation flexible, portable, and wearable miniaturized electronic devices.

\section{Methods}

Materials. The layered ternary carbide $\left(\mathrm{Ti}_{3} \mathrm{AlC}_{2}\right)$ MAX-phase powders were purchased from Carbon-Ukraine Ltd. (particle size $<200 \mu \mathrm{m}$, Ukraine). The chemicals, including hydrochloric acid $(\mathrm{HCl})$, lithium fluoride ( $\mathrm{LiF})$, ammonium chloride $\left(\mathrm{NH}_{4} \mathrm{Cl}\right)$, and ammonium hydroxide $\left(\mathrm{NH}_{4} \mathrm{OH}\right)$, were purchased from Sigma-Aldrich Co (St. Louis, MO, USA). Deionized water (DIW) was obtained using a water-purification system (Direct Q3) purchased from Millipore (Bedford, MA, USA).

Synthesis of the $\mathbf{T i}_{\mathbf{3}} \mathbf{C}_{\mathbf{2}} \mathbf{T}_{\mathbf{x}} \mathbf{M X e n e}$. The $\mathrm{Ti}_{3} \mathrm{C}_{2} \mathrm{~T}_{x}$ MXene was obtained from $\mathrm{Ti}_{3} \mathrm{AlC}_{2}$ precursors by modifying a previously reported method ${ }^{25}$. A quantity of $2 \mathrm{~g}$ of $\mathrm{LiF}$ was dissolved into $40 \mathrm{~mL}$ of a $9 \mathrm{M} \mathrm{HCl}$ solution in a reactor. The solution was stirred for $30 \mathrm{~min}$ at $35^{\circ} \mathrm{C}$. After slowly adding $2 \mathrm{~g}$ of the $\mathrm{Ti}_{3} \mathrm{AlC}_{2}$ (MAX phase) 
powder, the mixture was stirred in an argon atmosphere for $24 \mathrm{~h}$. The $\mathrm{Al}$ layer in the MAX phase was etched to exfoliate the $\mathrm{Ti}_{3} \mathrm{C}_{2} \mathrm{~T}_{x}$. Then, $40 \mathrm{~mL}$ of the obtained solution was divided into $20 \mathrm{~mL}$ conical tubes and diluted with water $(20 \mathrm{~mL})$. To separate the MXene dispersion from the acid, it was washed with water using a centrifuge until the $\mathrm{pH}$ reached 6. As the $\mathrm{pH}$ of the solution was almost neutral, the MXene in the dispersion did not sink well through centrifugation due to an increase in the negative zeta potential of their surface. The washed solution $(\mathrm{pH} 6)$ was centrifuged repeatedly to purify the MXene sheets and to concentrate the MXene dispersion. The obtained solution was sealed with parafilm and stored at $\sim 5^{\circ} \mathrm{C}$.

Wet-spinning of the pure $\mathbf{T i}_{3} \mathbf{C}_{\mathbf{2}} \mathbf{T}_{\mathbf{x}}$ (All-MXene) fibers. The MXene dispersion was placed in a syringe and extruded through the nozzle (diameter $=210 \mu \mathrm{m}$ ) into the prepared coagulant. The coagulate solution was a mixture of $\mathrm{NH}_{4} \mathrm{Cl}(50 \mathrm{~g})$, $\mathrm{NH}_{4} \mathrm{OH}$ solution $(20 \mathrm{~mL})$, and DIW $(1000 \mathrm{~mL})$. The MXene dispersions were extruded at a velocity of $7 \mathrm{~mL} \mathrm{~h}^{-1}$. The extruded MXene fibers in the coagulate solution were transferred to a washing bath by rollers. The washed fibers were dried in air and then stored in a dry chamber.

Characterization. The concentration of the concentrated dispersion was defined by cross-validation with the Beer-Lambert Law using UV-vis spectroscopy (Lambda 650S, Perkin Elmer, USA) and directly measuring the mass of the powder per unit volume of dispersion using an ultra-micro balance (XPR2U, MettlerToledo GmbH, Greifensee, Switzerland). The dimensions of the MXene sheets and the morphologies of the MXene fibers were characterized using SEM (S4800, Hitachi, Japan) at $15 \mathrm{kV}$ and $10 \mu \mathrm{A}$ without Pt sputtering. The topography of the MXene sheets was observed via AFM (XE-70, Park Systems, Korea) in tapping mode. The measured data were processed using a data processing and analysis software (XEI, Park Systems). The topography and a current image of the MXene nanosheets on the Si wafers were obtained simultaneously during the C-AFM scan. In this study, we conducted current measurements on the in-plane surface, which was consistent with the direction of the carrier transport parameter measurements. A cantilever (CDT-CONTR, Park systems) was used, and the measurement was performed with a fixed bias of $10 \mathrm{~V}$. The cantilever had a resonance frequency of $\sim 20 \mathrm{kHz}$, a spring constant of $0.5 \mathrm{~N} \mathrm{~m}^{-1}$, and a tip radius between 100 and $200 \mathrm{~nm}$. All measurements were performed at room temperature $\left(\sim 25^{\circ} \mathrm{C}\right)$ and in ambient conditions. The synthesized MXene single layer was characterized using HR-TEM (JEM-2100F, JEOL, Japan). The terminal group and the chemical state of the MXene were analyzed via XPS (Theta probe, Thermo Scientific, UK) with monochromatic $\mathrm{Al} \mathrm{Ka}$ radiation. The XPS spectra were analyzed using Xpspeak41 software. The rheological properties of the MXene dispersion were measured using a rheometer (MCR 501, Anton Paar, Austria) under both steady shear and dynamic oscillatory conditions. The viscoelastic properties of the $\mathrm{Ti}_{3} \mathrm{C}_{2} \mathrm{~T}_{x}$ dispersion were investigated by measuring the storage and loss modulus as a function of frequency from 0.1 to 1000 $\mathrm{rad} / \mathrm{s}$. During the frequency sweep, the strain amplitude was maintained at $0.1 \%$ (a gap of $\left.1 \mathrm{~mm}, 25^{\circ} \mathrm{C}\right)^{35}$. An optical image of the meter-long MXene fibers with a ruler was obtained by joining several close-up images taken vertically from above the fibers because the fibers were too thin for an image to be obtained from far away. The tensile properties of the MXene fibers prepared from different concentrations of dispersion were investigated using a universal testing machine (5966, Instron, USA) equipped with a $10 \mathrm{~N}$ load cell that operated at a crosshead speed of $2.5 \mathrm{~mm} \mathrm{~min}^{-1}$ and had a gauge length of $25 \mathrm{~mm}$; the tensile measurements of single ultrafine fibers reported in a previous study were used as a reference ${ }^{14}$. The fibers were loaded on a specific rectangular frame. The mechanical strength of the MXene fibers was calculated by dividing the force by the cross-sectional area. The electrical conductivity of the MXene fibers was measured using a multimeter (DMM 7510 1/2, Keithley Instruments, USA) via the four-point probe method. Four electrodes were separated by a distance of 0.4 $\mathrm{mm}$. Since the conductivity of MXene fibers is highly affected by the atmospheric humidity, the measurement was made in a dry chamber. The conductivity $(\rho)$ of a single fiber was calculated according to Eq. (1) as follows ${ }^{46}$ :

$$
\rho=\frac{\pi d^{2} R}{4 L}
$$

where $d$ is the diameter, $R$ is the electrical resistance, and $L$ is the length of the fiber. The diameter of the fiber was calculated based on the cross-sectional area measured in the scanning electron microscope (Supplementary Fig. 7).

Prediction of phase change using the Onsager model. To predict the critical value of the anisotropic phase, we measured the distribution of the MXene monolayer lateral sizes (Supplementary Fig. S1c) and found a reasonable agreement with the Onsager model for lyotropic liquid crystals in a disk formulation. The concentration (mass fraction) at the isotropic-nematic phase transition ( $C$, the critical value) was calculated according to Eq. (2) as follows ${ }^{33}$ :

$$
\frac{d}{I} \approx 5 \frac{\rho_{\text {MXene }}}{\rho_{\text {suspension }}} C^{-1}
$$

where $d$ and $l$ are the lateral size and thickness of the MXene nanosheets, respectively; $\rho_{\text {MXene }}$ is the true material density $\left(5.2 \mathrm{~g} \mathrm{~cm}^{3}\right)^{47}$; and $C$ is the concentration (mass fraction) at the isotropic-nematic phase transition.

\section{Data availability}

The datasets generated during and/or analyzed during the current study are available from the corresponding author on reasonable request. Source data are provided with this paper.

Received: 14 November 2019; Accepted: 11 May 2020;

Published online: 04 June 2020

\section{References}

1. Compton, O. C. \& Nguyen, S. T. Graphene oxide, highly reduced graphene oxide, and graphene: versatile building blocks for carbon-based materials. Small 6, 711-723 (2010).

2. Mas-Balleste, R., Gomez-Navarro, C., Gomez-Herrero, J. \& Zamora, F. 2D materials: to graphene and beyond. Nanoscale 3, 20-30 (2011).

3. Liu, J. et al. Hydrophobic, flexible, and lightweight MXene foams for high performance electromagnetic-interference shielding. Adv. Mater. 29, 1702364 (2017).

4. Kurra, N., Ahmed, B., Gogotsi, Y. \& Alshareef, H. N. MXene-on-paper coplanar microsupercapacitors. Adv. Energy Mater. 6, 1601372 (2016).

5. Dong, Z. et al. Facile fabrication of light, flexible and multifunctional graphene fibers. Adv. Mater. 24, 1856-1861 (2012).

6. Chen, C. et al. Functionalized boron nitride membranes with ultrafast solvent transport performance for molecular separation. Nat. Commun. 9, 1902 (2018).

7. Xia, P., Zhu, B., Yu, J., Cao, S. \& Jaroniec, M. Ultra-thin nanosheet assemblies of graphitic carbon nitride for enhanced photocatalytic $\mathrm{CO} 2$ reduction. J. Mater. Chem. A 5, 3230-3238 (2017).

8. Wang, H., Feng, H. \& Li, J. Graphene and graphene-like layered transition metal dichalcogenides in energy conversion and storage. Small 10, 2165-2181 (2014).

9. Zhu, W. et al. Black phosphorus flexible thin film transistors at gigahertz frequencies. Nano Lett. 16, 2301-2306 (2016).

10. Sun, Z. et al. Generalized self-assembly of scalable two-dimensional transition metal oxide nanosheets. Nat. Commun. 5, 3813 (2014).

11. Park, H. et al. Dynamic assembly of liquid crystalline graphene oxide gel fibers for ion transport. Sci. Adv. 4, eaau2104 (2018).

12. Aboutalebi, S. H. et al. High-performance multifunctional graphene yarns: toward wearable all-carbon energy storage textiles. ACS Nano 8, 2456-2466 (2014).

13. Meng, Y. et al. All-graphene core-sheath microfibers for all-solid-state, stretchable fibriform supercapacitors and wearable electronic textiles. Adv. Mater. 25, 2326-2331 (2013).

14. Eom, W. et al. Strengthening and stiffening graphene oxide fiber with trivalent metal ion binders. Part. Part. Syst. Charact. 34, 1600401 (2017).

15. Lukatskaya, M. R. et al. Ultra-high-rate pseudocapacitive energy storage in two-dimensional transition metal carbides. Nat. Energy 2, 17105 (2017).

16. Naguib, M. et al. Two-dimensional nanocrystals produced by exfoliation of Ti3AlC2. Adv. Mater. 23, 4248-4253 (2011).

17. Hantanasirisakul, K. et al. Fabrication of $\mathrm{Ti}_{3} \mathrm{C}_{2} \mathrm{~T}_{\mathrm{x}}$ MXene transparent thin films with tunable optoelectronic properties. Adv. Electron. Mater. 2, 1600050 (2016).

18. Peng, Y.-Y. et al. All-MXene (2D titanium carbide) solid-state Microsupercapacitors for on-chip energy storage. Energy Environ. Sci. 9, 2847-2854 (2016).

19. Yang, W. et al. 3D Printing of freestanding MXene architectures for currentcollector-free supercapacitors. Adv. Mater. 31, 1902725 (2019).

20. Jiang, C. et al. All-electrospun flexible triboelectric nanogenerator based on metallic MXene nanosheets. Nano Energy 59, 268-276 (2019).

21. Seyedin, S., Yanza, E. R. S. \& Razal, J. M. Knittable energy storing fiber with high volumetric performance made from predominantly MXene nanosheets. J. Mater. Chem. A 5, 24076-24082 (2017).

22. Yang, Q. et al. MXene/graphene hybrid fibers for high performance flexible supercapacitors. J. Mater. Chem. A 5, 22113-22119 (2017).

23. Wang, Z. et al. High-performance biscrolled MXene/carbon nanotube yarn supercapacitors. Small 14, 1802225 (2018).

24. Zhang, J. et al. Highly conductive $\mathrm{Ti}_{3} \mathrm{C}_{2} \mathrm{~T}_{\mathrm{x}}$ MXene hybrid fibers for flexible and elastic fiber-shaped supercapacitors. Small 15, 1804732 (2019).

25. Alhabeb, M. et al. Guidelines for synthesis and processing of twodimensional titanium carbide $\left(\mathrm{Ti}_{3} \mathrm{C}_{2} \mathrm{~T}_{\mathrm{x}}\right.$ MXene). Chem. Mater. 29, 7633-7644 (2017).

26. Lipatov, A. et al. Elastic properties of $2 \mathrm{D} \mathrm{Ti}_{3} \mathrm{C}_{2} \mathrm{~T}_{\mathrm{x}}$ MXene monolayers and bilayers. Sci. Adv. 4, eaat0491 (2018).

27. Liu, F. et al. Preparation of $\mathrm{Ti}_{3} \mathrm{C}_{2}$ and $\mathrm{Ti}_{2} \mathrm{C}$ MXenes by fluoride salts etching and methane adsorptive properties. Appl. Surf. Sci. 416, 781-789 (2017). 
28. Maleski, K., Mochalin, V. N. \& Gogotsi, Y. Dispersions of two-dimensional titanium carbide MXene in organic solvents. Chem. Mater. 29, 1632-1640 (2017).

29. $\mathrm{Li}$, Y. et al. Facile preparation of in situ coated $\mathrm{Ti}_{3} \mathrm{C}_{2} \mathrm{~T}_{\mathrm{x}} / \mathrm{Ni}_{0.5} \mathrm{Zn}_{0.5} \mathrm{Fe}_{2} \mathrm{O}_{4}$ composites and their electromagnetic performance. RSC Adv. 7, 24698-24708 (2017).

30. Kim, S. J. et al. Interfacial assembly of ultrathin, functional MXene films. ACS Appl. Mater. Interfaces 11, 32320-32327 (2019).

31. Choi, H., Seok Woo, J., Tark Han, J. \& Park, S.-Y. Fabrication of waterdispersible single-walled carbon nanotube powder using N-methylmorpholine N-oxide. Nanotechnology 28, 465706 (2017).

32. Xia, Y. et al. Thickness-independent capacitance of vertically aligned liquidcrystalline MXenes. Nature 557, 409-412 (2018).

33. Guo, F. et al. Hydration-responsive folding and unfolding in graphene oxide liquid crystal phases. ACS Nano 5, 8019-8025 (2011).

34. Morris, E. R., Cutler, A., Ross-Murphy, S., Rees, D. \& Price, J. Concentration and shear rate dependence of viscosity in random coil polysaccharide solutions. Carbohydr. Polym. 1, 5-21 (1981).

35. Akuzum, B. et al. Rheological characteristics of 2D titanium carbide (MXene) dispersions: a guide for processing MXenes. ACS Nano 12, 2685-2694 (2018).

36. Jalili, R. et al. Formation and processability of liquid crystalline dispersions of graphene oxide. Mater. Horiz. 1, 87-91 (2014).

37. Naficy, S. et al. Graphene oxide dispersions: tuning rheology to enable fabrication. Mater. Horiz. 1, 326-331 (2014).

38. Jalili, R. et al. Scalable one-step wet-spinning of graphene fibers and yarns from liquid crystalline dispersions of graphene oxide: towards multifunctional textiles. Adv. Funct. Mater. 23, 5345-5354 (2013).

39. Cong, H.-P. et al. Wet-spinning assembly of continuous, neat, and macroscopic graphene fibers. Sci. Rep. 2, 613 (2012).

40. Xu, Z. \& Chao, G. Graphene chiral liquid crystals and macroscopic assembled fibres. Nat. Commun. 2, 571 (2011).

41. Chen, L. et al. Toward high performance graphene fibers. Nanoscale 5, 5809-5815 (2013).

42. $\mathrm{Xu}, \mathrm{Z}$. et al. Ultrastrong fibers assembled from giant graphene oxide sheets Adv. Mater. 25, 188-193 (2013).

43. Xiang, C. et al. Large flake graphene oxide fibers with unconventional $100 \%$ knot efficiency and highly aligned small flake graphene oxide fibers. $A d v$. Mater. 25, 4592-4597 (2013).

44. Ma, T. et al. A bioinspired interface design for improving the strength and electrical conductivity of graphene-based fibers. Adv. Mater. 30, 1706435 (2018).

45. Ling, Z. et al. Flexible and conductive MXene films and nanocomposites with high capacitance. Proc. Natl Acad. Sci. USA 111, 16676-16681 (2014)

46. Rebouillat et al. Measuring the electrical conductivity of single fibres. Int. J. Electrochem. Sci. 6, 5731-5740 (2011).

47. Naguib et al. Two-dimensional transition metal carbides. ACS Nano 6 1322-1331 (2012).

\section{Acknowledgements}

The authors thank the Basic Science Research Program (2017R1A2B4010771 and 2016R1A6A1A03013422) of the National Research Foundation of Korea funded by the Ministry of Science, ICT \& Future Planning. The prepared samples were analyzed using TEM, XPS, and XRD instruments installed at the Hanyang LINC Analytical Equipment Center (Seoul).

\section{Author contributions}

Conceptualization, T.H.H.; methodology and investigation, W.E., H.S., R.B.A., S.H.L., K.H.L., D.J.K., and T.H.H.; supervision, T.H.H.; W.E., H.S., R.B.A., S.H.L., K.H.L., D.J.K. and T.H.H. contributed to the writing of, reading of, and commenting on the manuscript.

\section{Competing interests}

The authors declare no competing interests.

\section{Additional information}

Supplementary information is available for this paper at https://doi.org/10.1038/s41467020-16671-1.

Correspondence and requests for materials should be addressed to T.H.H.

Peer review information Nature Communications thanks Zong-Huai Liu and other, anonymous, reviewers for their contributions to the peer review of this work. Peer review reports are available.

Reprints and permission information is available at http://www.nature.com/reprints

Publisher's note Springer Nature remains neutral with regard to jurisdictional claims in published maps and institutional affiliations.

Open Access This article is licensed under a Creative Commons Attribution 4.0 International License, which permits use, sharing, adaptation, distribution and reproduction in any medium or format, as long as you give appropriate credit to the original author(s) and the source, provide a link to the Creative Commons license, and indicate if changes were made. The images or other third party material in this article are included in the article's Creative Commons license, unless indicated otherwise in a credit line to the material. If material is not included in the article's Creative Commons license and your intended use is not permitted by statutory regulation or exceeds the permitted use, you will need to obtain permission directly from the copyright holder. To view a copy of this license, visit http://creativecommons.org/ licenses/by/4.0/.

(C) The Author(s) 2020 